\title{
Quantum dynamics in the smallest water droplet
}

Perspective on "Concerted Hydrogen-Bond Breaking by Quantum

Tunneling in the Water Hexamer Prism" by Richardson et al

David C. Clary

Department of Physical and Theoretical Chemistry

University of Oxford

South Parks Road

Oxford OX1 3QZ, UK

david.clary@chem.ox.ac.uk

A new type of concerted hydrogen bond motion is discovered in an experimental and quantum theory study of a water cluster.

Understanding water is central to so many scientific areas ranging from geology, to astronomy to biology. In its most fundamental form this first requires studying the properties of size-selected clusters of water molecules $\left(\mathrm{H}_{2} \mathrm{O}\right)_{n}$ in which the $n$ individual water molecules are held together by hydrogen bonds. Developing an accurate potential surface for $\left(\mathrm{H}_{2} \mathrm{O}\right)_{n}$ as $n$ increases will lead to a highly reliable potential function for liquid water and ice which will have a huge number of applications in simulations across the sciences (1). The water hexamer $\left(\mathrm{H}_{2} \mathrm{O}\right)_{6}$ has received much study as it is the smallest cluster to have a three dimensional geometry and has been dubbed the "smallest water droplet" (2). Advanced spectroscopic methods have been developed and applied to provide experimental information on its structure and properties (3). This has been supported by theory both on the development of accurate potential energy surfaces for the interaction of water molecules (1) and also in the development of quantum dynamical methods for simulating the motion of the water molecules and calculating the spectral observables (4). On page yy of this issue (5) Richardson et al describe experimental and theoretical evidence for a new type of motion in water clusters in which two hydrogen bonds are broken simultaneously in the prism form of $\left(\mathrm{H}_{2} \mathrm{O}\right)_{6}$. 
Until quite recently the most precise experimental information on the structures of small water clusters came from studies in which the intramolecular vibrational modes were probed in the far-infrared (terahertz) region of the spectrum (3). Very recently it has become possible to study the rotational motion of these clusters directly in the microwave region by using a broadband Fourier Transform technique (6). This has allowed for the new experimental developments reported in this issue (5).

A water cluster such as $\left(\mathrm{H}_{2} \mathrm{O}\right)_{6}$ can have several different forms which take the shapes of books, cages and prisms (2). Within these forms there are many equivalent positions which correspond to minima on the potential surface without any breaking of covalent bonds. Between equivalent minima there is an energy barrier and thus rearrangements can occur via quantum tunneling. This feature leads to splittings of lines in the observed microwave spectrum, an effect which has been known for over 60 years in fluxional molecules such as $\mathrm{NH}_{3}$ (7). Thus observations of the tunneling patterns in the microwave spectra for $\left(\mathrm{H}_{2} \mathrm{O}\right)_{n}$ provide exquisite information on the quantum dynamics of the hydrogen bond motion in the cluster. Furthermore, measuring the spectra corresponding to mixtures of different isotopes of oxygen ${ }^{16} \mathrm{O}$ and ${ }^{18} \mathrm{O}$ in $\left(\mathrm{H}_{2} \mathrm{O}\right)_{6}$ allows for a detailed interpretation of the tunneling dynamics (6).

To understand these spectra it is therefore essential to use a quantum dynamical theory. This remains a real challenge for a system such as $\left(\mathrm{H}_{2} \mathrm{O}\right)_{6}$ which has 54 dimensions. However, clever adaptation of Feynman's Path Integral theory (8) has provided a quantum dynamical method readily capable of calculating these tunneling spectra from the best available potential energy surfaces (4). This "ring polymer" molecular dynamics (RPMD) method exploits the mapping onto the quantum mechanical problem of an infinite number of copies of the system coupled by harmonic springs (9). It is found that a relatively small number of copies are needed to converge results for water clusters and the system can be simulated using classical molecular dynamics which is computationally inexpensive and readily applicable to many dimensions. The RPMD method of simulation was first mainly applied to the study of chemical reaction rates (9) but the technique can be adapted to calculate the tunneling splittings in the spectra of molecular clusters (4). 


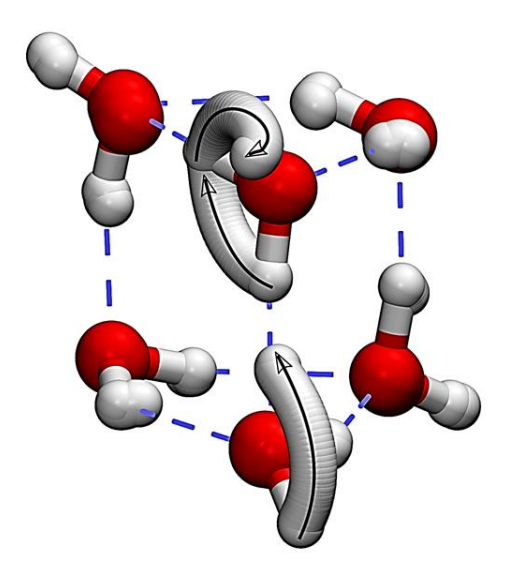

Concerted breaking of two hydrogen bonds in the prism form of the water hexamer (Figure adapted from reference 5).

Previously, theory had identified hydrogen bond rearrangements in water clusters which were either flips, with no hydrogen bond breaking, or bifurcations where one bond breaks (2). The new finding is that the hydrogen bond dynamics in the prism form of $\left(\mathrm{H}_{2} \mathrm{O}\right)_{6}$ can involve the simultaneous breaking of two hydrogen bonds as shown in the figure above. This novel mechanism has significant implications not only for the study of water clusters but also for the numerous systems where water molecules play a role in confined environments. In addition, the excellent detailed agreement between the first-principles calculations and experiment on $\left(\mathrm{H}_{2} \mathrm{O}\right)_{6}$ demonstrates that theory is getting much closer to a highly accurate description of water and, through this process, to providing a more detailed quantitative understanding of hydrogen bond dynamics.

\section{References}

1. V. Babin, G. R Medders, F. Paesani, J Phys. Chem. Lett. 3, 3765 (2012).

2. K. Liu et. al., Nature 381, 501 (1996).

3. F. N. Keutsch, R. J. Saykally, Proc. Natl. Acad. Sci. USA 98, 10533 (2001).

4. J. O. Richardson, S. C. Althorpe, D. J. Wales, J. Chem. Phys., 135, 124109 (2011).

5. J. O. Richardson et. al., Science xx,yy, (2016)

6. C Pérez, et. al., Science 336, 897 (2012).

7. C. Townes and A. Schawlow, Microwave Spectroscopy, (McGraw-Hill, New York, 1955).

8. R.P. Feynman, A. R. Hibbs and D. F. Styer, Quantum mechanics and path integrals, Mineola, N.Y.; Dover (2010).

9. I. R. Craig, D. E. Manolopoulos, J. Chem. Phys. 122, 084106 (2005). 